\title{
Estimation of Energy Consumption when Wearing Power Assist Suits
}

\author{
Toshitake Araie, Uichi Nishizawa, Tomozumi Ikeda, Akira Kakimoto, and Shigeki Toyama
}

\begin{abstract}
Agricultural power assist suits (PAS) have been developed and researched in our laboratory. In this study, the performance of a PAS is evaluated without burdening subjects through kinetic simulation. According to the formula for deriving energy consumption, the ratio of the energy consumption to the joint torque is same. Therefore, the joint torque of the knee is derived to investigate the influence of kinetic simulation the on femur. According to kinetic simulation, a PAS can reduce energy consumption. Further, the performance of PAS can be extracted by adjusting size. The validity of simulation is evaluated by comparing with other results. The simulation results and dynamics calculations agree well, but the actual measurements of some subjects show differences. The factors considered are the measurement error of the joint angle, difference in the position for pasting the electrode to measure the myopotential, and the asymmetry of muscle tension. Differences with considerable errors were observed for some test subjects in comparison with actual values, but a good match was observed for the case in which the PAS was worn for those test subjects that also showed a good match for the case without the PAS.
\end{abstract}

Index Terms-Dynamics calculations, energy consumption, joint torque, Kinetic simulation, Myopotential, power assist suit.

\section{INTRODUCTION}

A considerable amount of time has passed since aging population and declining birth rates have become a serious issue in Japan. The effect of aging has been particularly significant in agriculture, with $66.3 \%$ of the agricultural workers aged 65 and over as of 2017. In addition, many of the tasks involved in agriculture are physically demanding. Efforts are being made to reduce burden in agricultural work and improve efficiency by introducing farm machines. However, the introduction of such machines may be difficult in some areas owing to the geographical conditions of the farmland, in which case burdensome work continues to remain a requirement. In view of this background, many institutions are researching on assist suits that agricultural workers can wear to reduce their workload. Examples of these include the Raku Vest ARM-1 developed by Kubota Corporation and the WAS-LiBERo [1], [2] developed at Wakayama University.

This university laboratory developed and evaluated the power assist suit (PAS) for farm work [3]-[7].

A PAS is an equipment that reduces the physical burden of

Manuscript received November 30, 2017; revised April 1, 2018.

T. Araie, T. Ikeda, and A. Kakimoto are with the Polytechnic University of Japan, 2-32-1, Ogawa-nishimachi, Kodaira-shi, Tokyo, Japan (e-mail: araie@uitec.ac.jp; ikeda@uitec.ac.jp; kakimoto@uitec.ac.jp).

U. Nishizawa and S. Toyama are with the Tokyo University of Agriculture and Technology, 2-24-16, Nakacho, Koganei, Tokyo, Japan (e-mail: n-uichi@cc.tuat.ac.jp; toyama@cc.tuat.ac.jp). farm work for the person wearing the suit. Therefore, it is crucial that the impact of the PAS on the person wearing it is investigated to analyze its performance. Common approaches to measuring impacts on human bodies include respiratory metabolism and myopotential [8]-[10]. However, determining local impacts is difficult with respiratory metabolism. Moreover, the treatment of myopotential data can be a challenge owing to the large influence of noise at a low voltage. Further, field measurements require motions that simulate farm work performed by test subjects, which impose physical burden on the subjects. From an ethical perspective, measurements need to be taken by considering the physical burden on test subjects, and there are limits to the number of experiments that can be conducted at a time. Therefore, this study proposes a method of quantitatively evaluating the PAS performance through a kinetic simulation and evaluates the performance of the PAS through the proposed method.

\section{Agricultural PAS}

The Agricultural PAS used in this study was developed to reduce the workload for agricultural workers cultivating grapes, Asian pears, and other shelf crops. The suits maintains the agricultural worker's stance by spring or ratchet mechanisms without the use of motors or batteries Fig. 1. shows an outer view of the Agricultural PAS used in the tests. The suits assist in light work by maintaining the arms that handle the load in shelf crop harvesting in a raised stance and the hips in a half-sitting posture. The suit is structured such that the wearer does not feel the weight of the unit while in a standing or half-sitting posture. The design standard was set close to the average build of a male Japanese person with a height of $170 \mathrm{~cm}$ and a weight of $65 \mathrm{~kg}$. The frame position of each part can be adjusted to change the size.
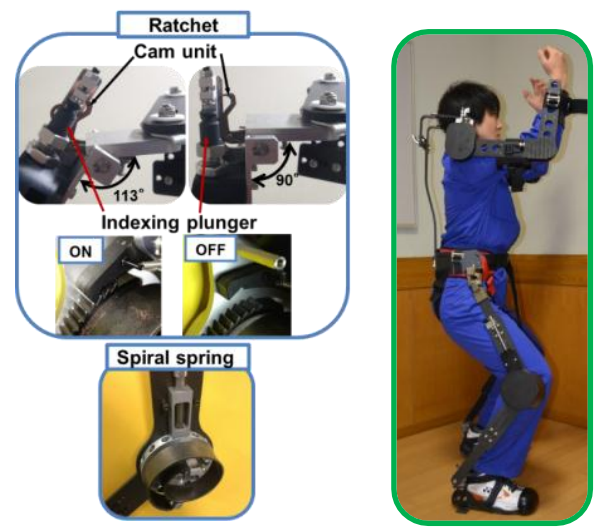

Fig. 1. Aricultural power asist suit. 


\section{PERformance Evaluation Method}

\section{A. Energy Consumption by Muscles}

This study evaluates the performance of the PAS considering the changes in energy consumption by muscles while the suit is being worn. For estimation of energy consumption, Ehara et al. [11] have proposed an equation of derivation. The following equation is obtained by reorganizing the variables and constants of the equation of derivation:

$$
P=f\left\{v+\frac{\left(v+0.9 L_{0}\right)^{2}}{3.6 L_{0}-v}\right\} \quad(v \geqq 0) .
$$

where, $P$ is the energy consumed by a muscle per unit time, $\mathrm{f}$ is the muscle tension, $\mathrm{v}$ is the velocity of muscle contraction, and $\mathrm{L}_{0}$ is the length of the static muscle.

The mechanism of the PAS is designed mainly to assist retention of a posture. Muscle length does not change while a posture is retained, and therefore $v=0$. Thus, equation (1) becomes

$$
P=0.225 f L_{0}
$$

\section{B. Performance Evaluation Index}

Joint torque $M i$ for muscle tension $\mathrm{f}$ of a given muscle $i$ is expressed as follows.

$$
M_{i}=f \times d
$$

where $d$ is the moment arm length used when converting muscle tension into a joint torque. When the same test subject takes the same position, the value of $d$ for each muscle becomes the same irrespective of whether the subject is wearing the PAS. Joint torque $M$ around a given joint is the sum of the torques exerted by the muscle involved in generating the torque. Assume that $M$ can be combined as

$$
M=\sum M_{i}=F \times D
$$

In the equation, $F$ is the resultant force of the muscle tensions and $D$ is the moment arm length after the torques are combined. $D$ is constant for the same position and same test subject. Therefore, taking the ratio of energy consumption $P_{P A S}$ when the PAS is worn and energy consumption $P_{N P A S}$ when the PAS is not worn, the following equation is obtained from equations (2) and (4):

$$
\frac{P_{P A S}}{P_{N P A S}}=\frac{f_{P A S}}{f_{N P A S}}=\frac{M_{P A S}}{M_{N P A S}} .
$$

Based on the above explanation, energy consumption can be compared with the joint torque.

Additionally, as a performance index for the PAS, reduction rate $R$ with respect to energy consumption is defined as follows, considering equation (5):

$$
R=\left(1-\frac{P_{P A S}}{P_{N P A S}}\right) \times 100=\left(1-\frac{M_{P A S}}{M_{N P A S}}\right) \times 100
$$

where, the absolute value of $R$ is the amount of change in energy consumption by wearing the PAS; the load is reduced if $R>0$ and is increased if $R<0$. By calculating $R$, this study performs a quantitative evaluation of the assist performance of the PAS.

\section{COMPARISON OF ENERGY CONSUMPTION VIA SIMULATION}

\section{A. Simulation Overview}

The PAS has an assist mechanism that uses a spiral spring for the legs to support a half-rising position. To investigate the changes in the burden to the femur in the presence of leg assist mechanism, the knee joint torque with and without the PAS was derived for a half-rising position; the energy consumption for both cases was compared. A kinetic simulation was performed to derive knee joint torque.

The case in which the PAS is not worn was simulated by first developing a simulation model of the human body and PAS separately and then performing a simulation with the human model alone. The case in which the PAS is worn was simulated by equipping the PAS model onto the human body model. Fig. 2. shows the model with and without the PAS.

For obtaining the physical property values of the human model, an arbitrary test subject (a male in his 20s) was chosen, and the measured lengths of each of his body parts were used in the model. The measured body parts included the feet, crus, femur, torso, head, upper arm, forearm, hands, the distance between hip joints, and the distance between shoulder joints. The mass of each body part was calculated by using the measured values and an equation of derivation obtained from a previous study [12]. For the physical property values of the PAS model, the values from the blueprint were used for the dimensions, and those of the dimensions and density of constituent materials were applied for the mass.

After setting the physical properties for the model, an arbitrary angle was set for the ankle, knee, and hip joints of

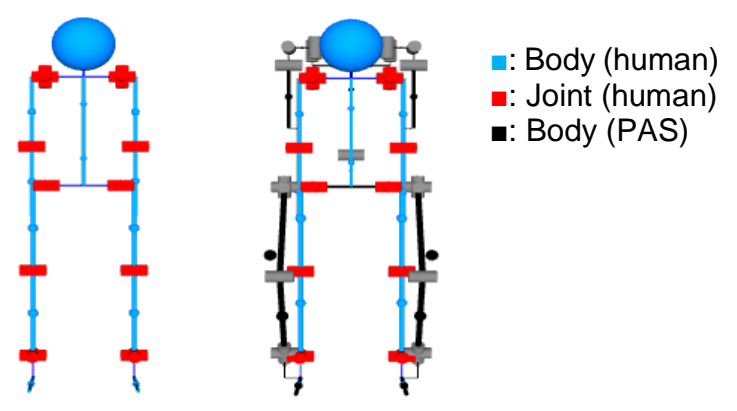

Fig. 2. Simulation model.

the human body to simulate the half-rising position. The angles set for each joint are given in Table I. The mean value of the knee joint torque obtained from the simulation performed using the human body model was used as the knee joint torque in a half-rising position, and the energy consumption was compared with this torque. 
TABLE I: JOINT ANGLES

\begin{tabular}{cc}
\hline \hline Joint & Angle $\left({ }^{\circ}\right)$ \\
\hline Ankle & 70 \\
Knee & 120 \\
Hip & 140 \\
\hline \hline
\end{tabular}

\section{B. Comparison of Results and Discussion}

To provide safety to the person wearing the PAS while walking, the knee joints in the legs of PAS do not extend completely even in an upright position and their angle remains below $180^{\circ}$. Therefore, this study compared two cases: one with the PAS and the half-rising position is taken after turning on the switch of the leg assist mechanism in a normal upright position, and another wherein the PAS provides "maximum assistance" in which the half-rising position is taken after turning on the switch for leg assist mechanism once the angles of knee joints of the PAS were forcefully set to $180^{\circ}$.

Fig. 3. shows the knee joint torque values under each condition. Fig. 4. shows reduction rate $R$ for the energy consumption. It can be observed in Fig. 3. that the burden on the femur is reduced by the PAS assist mechanism as the knee joint torque is reduced. However, $R$ in Fig. 4 . is approximately $22 \%$ with the PAS and approximately $67 \%$ with maximum assistance, which is approximately thrice the value with the PAS. One of the causes is the property of the spiral spring owing to the form of the PAS legs in an upright position.

The spiral spring maintains its natural length before the leg assist mechanism is turned on and interferes with the knee joints of the PAS once the switch is turned on and creates a repulsive force by deforming according to the changes in the joint angles.

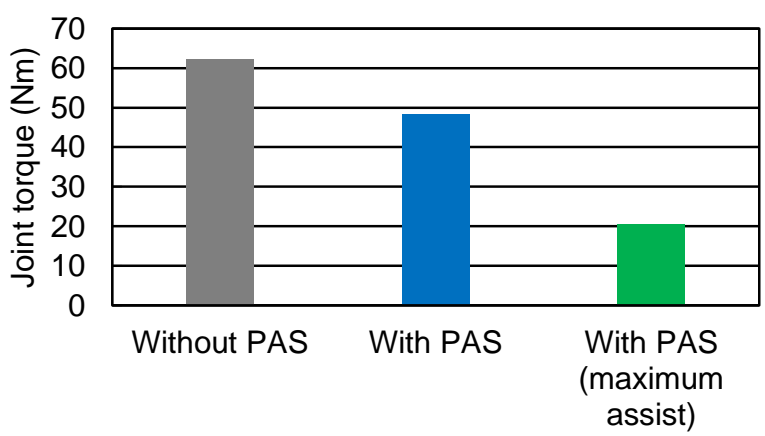

Fig. 3. Joint torque of knee.

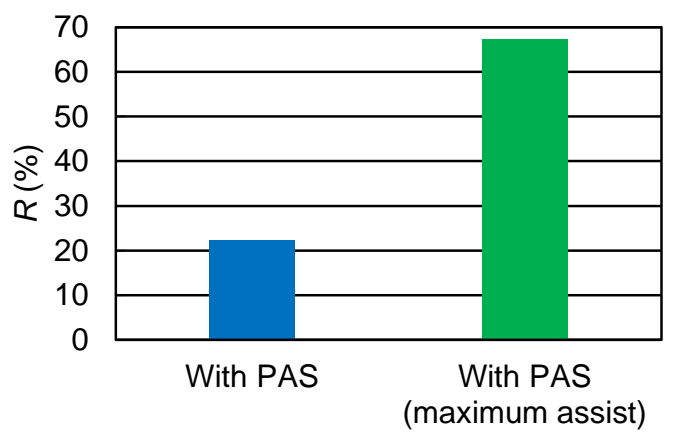

Fig. 4. Reduction rate of energy consumption.

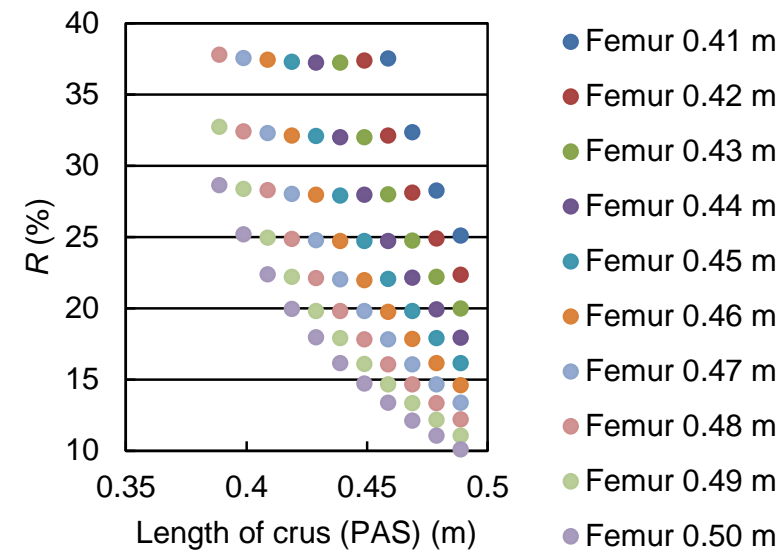

Fig. 5. Comparison of reduction rate.

In other words, the repulsive force of the spiral spring is proportional to the difference between the knee joint angle of the PAS at the time the switch is turned on and the knee joint angle of the PAS in a half-rising position. A difference in $R$ was observed as the assist force was reduced because of the reduced change in angle of the knee joints of the PAS on bending from an upright position to a half-rising position.

The knee joint angle of the PAS in an upright position can be changed by adjusting the size of the PAS legs. Therefore, the relationship between the size of PAS legs and energy consumption in the femur was investigated. The leg size of the PAS was varied within a range in which the sum of the total length of the crus and femur of PAS did not exceed the sum of the total length of the crus and femur of the test subject. The knee joint torque in a half-rising position was derived once more and $R$ was compared. Fig. 5. shows the comparison results. $R$ depended heavily on the sum of the total length of the crus and femur, and the value increased as it neared the sum of the total length of the crus and femur of the test subject. This was because the knee joint of the PAS in an upright position extended and reached closer to $180^{\circ}$ on setting the total length of the PAS leg closer to the total length of the leg of the test subject; the change in the angle increased as PAS moved from an upright position to a half-rising position.

\section{EVALUATION OF THE VALIDITY OF SIMULATION}

\section{A. Definition of Compared Subjects for the Evaluation}

In this study, simulation results alone were used to derive the reduction rate of the energy consumption. Therefore, the validity of the simulation results needs to be discussed to justify the reduction rate of energy consumption. The simulation results were validated through a comparison with dynamics calculations and actual measurements.

The equation of derivation for the joint torque of both the knees at a half-rising position is shown below.

$$
\begin{aligned}
& 2 T_{\text {knee }}=2 m_{\text {femur }} g\left(l_{\text {femur }}^{\prime} G_{\text {femur }}\right)+m_{\text {body }} g\left(l_{\text {femur }}^{\prime} l_{\text {body }}^{\prime} G_{\text {body }}\right) \\
& +2 m_{\text {underarm }} g\left(l_{\text {femur }}^{\prime}-l_{\text {body }}^{\prime}-l_{\text {underarm }}^{\prime} G_{\text {underarm }}\right) \\
& +2 m_{\text {frontarm }} g\left(i_{\text {femur }}^{\prime}-l_{\text {body }}^{\prime} l_{\text {underarm }}^{\prime}+l_{\text {frontarm }}^{\prime} G_{\text {frontarm }}\right) .
\end{aligned}
$$




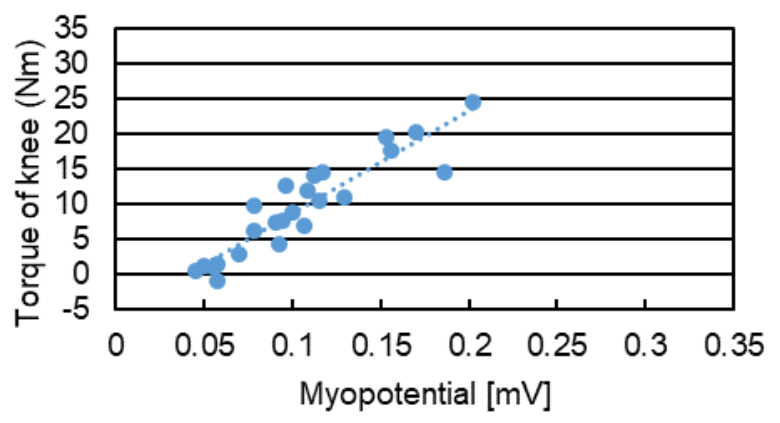

Fig. 6. Myopotential vs torque of knee.

where, $m$ is the mass of each body part, $g$ is the gravitational acceleration, $l$ ' is the vector component parallel to the ground at each body part, and $G$ is the location of the center of gravity for each body part. If the torques of both knees are symmetrical in a case where the posture is symmetrical between left and right, the knee joint torque per leg is derived by multiplying equation (7) with $1 / 2$.

As the joint torque does not appear as an external force when retaining a posture, it is difficult to directly measure the knee joint torque of a test subject in a half-rising position with measurement equipment. Therefore, the torque is derived using myopotential as the index of actual measurements. Myopotential can be obtained either through needle electromyography in which needle-like electrodes are pierced into a muscle, or a surface electromyogram in which myopotential is measured by attaching electrodes on the surface of the skin. This study utilized the surface electromyogram approach owing to a smaller burden on the test subjects. The method of deriving the joint torque from myopotential has been validated in an experiment conducted by Prilutsky et al [13]. The equation of derivation is shown below:

$$
M_{j}^{e}=\sum_{i}\left(\frac{E M G_{i \text { inst }}}{E M G_{i_{-} \max }}\right) F_{i_{-} \max } d_{i j} .
$$

where, $M_{j}^{e}$ is the torque of joint $\mathrm{j}, E M G_{i_{\text {inst }}}$ is the measured myopotential intensity of muscle $i$, and $E M G_{i_{\max }}$ is the myopotential intensity when the muscle tension of the muscle $i$ is at its maximum; $F_{i_{\max }}$ is the maximum value of the muscle tension, and $d_{i j}$ is the moment arm length used when converting muscle tension to a joint torque. Given that $E M G_{i_{\max }}, F_{i_{\max }}$, and $d_{i j}$ are the same for the same position of the same test subject, the equation shows that the myopotential intensity and joint torque are in a proportional relationship.

There are individual differences in the number of muscles per test subject. Therefore, the value of the proportional constant used to derive the joint torque is a different for each test subject. Considering this difference, the correlation between the torque and myopotential intensity was investigated empirically. A weight load was provided to the ankle joint of the test subject in a seated position, and the state in which the feet were afloat was maintained while keeping the knee joint fixed at an arbitrary angle. The myopotential of the femur was measured using electrodes stuck on the surface of the skin while maintaining the posture, and the angles of the hip and knee joints were measured using optical motion capturing. The measured myopotential was processed by rectification and smoothing and used as the myopotential intensity. The knee joint torque during the experiment was also derived using the measured values of the joint angles.

Fig. 6. shows the relationship between the knee joint torque and myopotential intensity when a weight is held. Taking the variation in data into account, linear approximation was performed.

The plot in which the knee joint torque was the vertical axis and the myopotential intensity was the horizontal axis was labeled "myopotential", and the plot in which myopotential intensity was the vertical axis. The joint torque in actual measurements was derived by substituting the myopotential intensity into the linear approximation.

\section{B. Comparison of results in a half-rising position}

The joint angle in a half-rising position and surface myopotential of the femur were measured. The joint angles were measured for the ankles, knees, hips, shoulders, and elbows from the sagittal plane. The position and muscle tension in the femur of the test subjects were assumed to be symmetrical, and measurements were taken only for the right side of the body. The knee joint torque was derived from the measured myopotential intensity, which was treated as the actual value. The measured joint angle was used in both the dynamics calculations and the simulation, and the torque was derived to ensure that the position would be the same for each method of derivation.

Fig. 7. shows the results without the PAS. Given that the errors between the results from the simulation and dynamics calculations were within a few $\%$, it can be said that nearly the same results were obtained from both the simulation and equation (7) for a scenario without the PAS. However, between the simulation and myopotential results, some cases resulted in a good match, as shown in (a), while other cases showed a significant difference as shown in (b), depending on the test subject.

The factors that caused such a result in the simulation included the measurement errors in the dimensions of the body and joint angles and the fact that masses were derived for each body part using the same equation of derivation for test subjects with different physiques. The influence of measurement errors in the joint angle was particularly large such that the knee joint torque changed by approximately $10 \%$ for a $1^{\circ}$ change in the ankle and knee joint angle, while the knee joint angle changed by approximately $5 \%$ for a $1^{\circ}$ change in the hip joint angle. The ratio that the mass of the femur, torso, and the head occupies in the mass of the human body is significant, and the position of the femur and torso likely had a large impact on the knee joint torque. The position of the femur in a half-rising position was determined by the joint angles of the ankles and knees, while the position of the torso was determined by the position of the femur and joint angle of the hips.

Therefore, the impact on the knee joint torque became greater than the ankle joint angle that affects the position of 


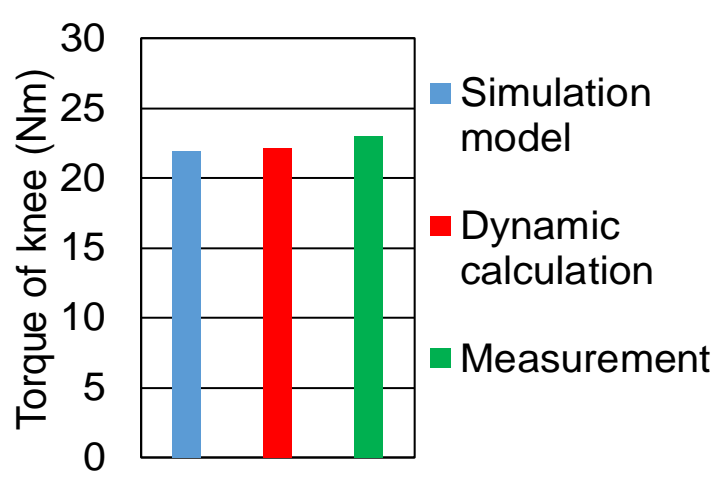

(a) Subject (agreement)

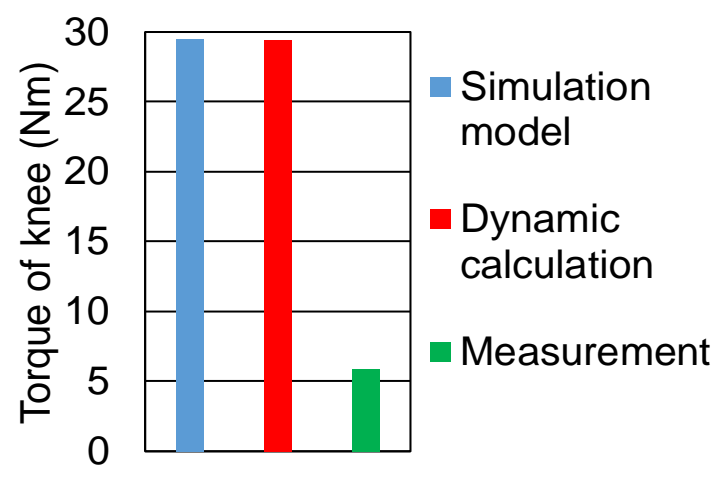

(b) Subject (disagreement)

Fig. 7. Comparison of joint torque without PAS

both the femur and torso as well as the hip joint angle in which the knee joint angle does not affect the femur position. On the other hand, factors that caused the results based on the myopotential shown in Fig. 7. include the fact that the muscle tension of the femurs were asymmetric in reality; further, the vastus intermedius could not be measured among the quadriceps, and the influence of the innervation zone due to an error in the location in which the measuring electrodes were placed.

Fig. 8. shows the results for the case with the PAS. The values of the dynamics calculations in the figure are values converted to a case without the PAS in which the effect of PAS is not considered. A good match was obtained between simulation results and actual measurements in the case with the PAS for test subjects who had a good match for the case without the PAS, as shown in (a), but test subjects who showed a considerable difference in the case without the PAS also showed a substantial difference in the case with the PAS, as shown in (b). A trend was also observed in which the values from the dynamics calculations were greater for the case with the PAS than the case without the PAS, which suggests that the PAS allows users to take a position that enhances their work efficiency.

\section{CONCLUSION}

The relationship between the joint torque and energy consumption of the human body was analyzed based on the calculation formula for deriving energy consumption from muscle tension; a method that used the reduction rate for

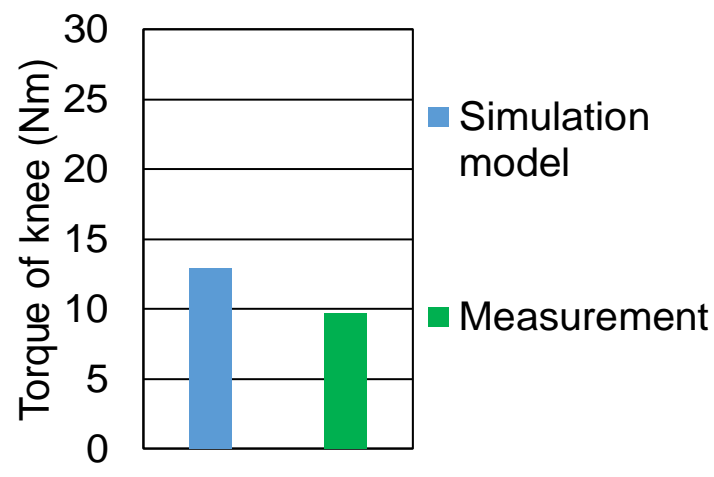

(a) Subject (agreement)

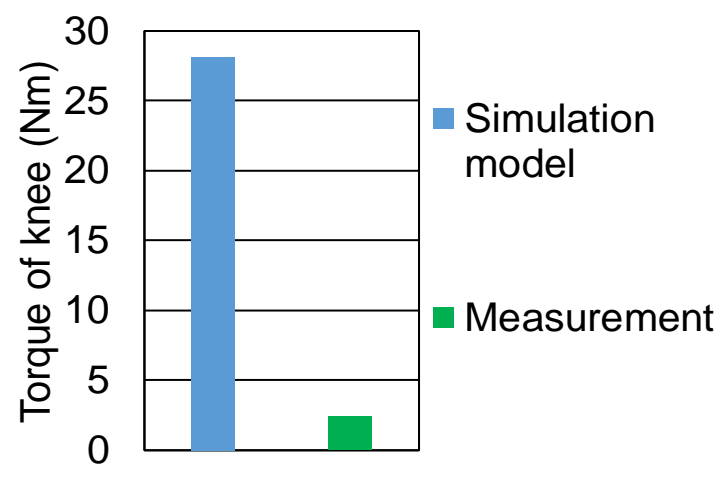

(b) Subject (disagreement)

Fig. 8. Comparison of joint torque with PAS.

energy consumption was proposed as an index for evaluating the performance of the PAS. The study showed that energy consumption can be reduced by wearing the PAS through deriving the reduction rate of energy consumption through a simulation; it was verified that the reduction rate could be improved by adjusting the size of the suit. To evaluate the validity of the developed simulation model, simulation results were compared with the actual values obtained using dynamics calculations and the myopotential intensity. This comparison verified that correct dynamics calculations were performed for the human body model not equipped with the PAS. However, differences with considerable errors were observed for some test subjects in comparison with actual values, but a good match was observed for the case in which the PAS was worn for those test subjects that also showed a good match for the case without the PAS.

Future challenges include reconsidering the method of measurement that would minimize the measurement error, which is thought to be the major cause of the difference between the measurement results and simulation results. While this study also performed an evaluation of the lower half of the PAS, the PAS developed in our university laboratory also has an assist mechanism for the upper body, which also needs to be investigated. Additionally, as this study could define an index for the evaluation of the PAS performance, another future challenge is to evaluate older PAS equipment developed in this university laboratory as well as PASs developed by other institutions to evaluate the improvements in PAS, while also discussing the pros and cons of the PASs. 


\section{REFERENCES}

[1] M. Sato and E. Yagi, "A study on power assist suit using pneumatic actuators based on calculated retaining torques for lift-up motion," Proceedings of SICE Annual Conference, pp. 628-632, 2011.

[2] K. Sano, E. Yagi and M. Sato, "Development of a wearable assist suit for walking and lifting-up motion using electric motors," Journal of Robotics and Mechatronics, vol. 25, no. 6, pp. 923-930, 2013.

[3] G. Yamamoto and S. Toyama, "Development of Wearable-Agri-Robot Mechanism for Agricultural Work ," International Conference on Intelligent Robots and System, pp. 5801-5806, 2009.

[4] S. Toyama and G. Yamamoto, "Wearable agrirobot," Journal of Vibroengineering, vol. 12, pp. 287-291, 2010.

[5] Y Y Nwe, et al., "Workload assessment with Ovako working posture analysis system (OWAS) in Japanese Vineyards with focus on pruning and berry thinning operations," J. Japan. Soc. Hort. Sci, vol. 81, no. 4 , pp. 320-326, 2012.

[6] T. Araie, T. Ikeda, U. Nishizawa, A. Kakimoto, S. Toyama, "Mechanism evaluation of agricultural power assist suit under development," Vibroengineering PROCEDIA, vol.8, pp.328-333, 2016.

[7] T. Araie, U. Nishizawa, T. Ikeda, A. Akira. S. Toyama, "Evaluation of Labor Burden Reduction Achieved through Wearing an Agricultural Power Assist Suit," International Journal of Modeling and Optimization, vol.7, no.4. pp.202-206, 2017.

[8] S. P. Arjunan, D. K. Kumar, and G. Naik, "Computation and evaluation of features of surface electromyogram to identify the force of muscle contraction and muscle fatigue," BioMed Research International, vol. 2014, 2014. http://dx.doi.org/10.1155/2014/197960.

[9] S. J. Strath, et al., "Evaluation of heart rate as a method for assessing moderate intensity physical activity," Medicine \& Science in Sports \& Exercise, vol. 32, pp. S465-S470, 2000.

[10] A. Tajima, "Assessment of energy expenditure: an association between heart rate and oxygen uptake in daily physical activity," International Journal of Analytical Bio-Science, vol. 2, no. 4, pp. 135-142, 2014.

[11] Y. Ehara, M. Beppu, S. Nomura, Y. Kunimi, "Estimation of energy consumption during level walking," Society of Biomechanisms Japan, vol.10, pp.163-172, 1990.

[12] Benno M. Nigg, Walter Herzog, Biomechanics of the Musclo-skeletal System, New Jersey: John Wiley \& Sons. p.459, 2007.

[13] Boris I. Prilutsky, Robert J. Greogor, "Analysis of muscle coordination strategies in cycling", IEEE transactions on rehabilitation engineering, vol. 8, Issue 3, pp. 362-370, 2000.

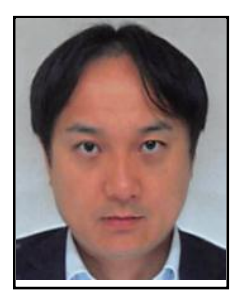

Toshitake Araie was born in Kaga of Japan, Sep. 19,1975 . He is 4rd year student in the Doctoral Program of Tokyo University of Agriculture and Technology. He is Assistant professor of faculty of human resources development at the Polytechnic University. His primary research interests include development of agricultural power assist suit, life support technology and embodied cognitive science.

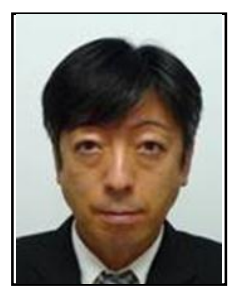

Uichi Nishizawa was born in Kodaira of Japan, 1970. He received his Ph.D degrees from the Tokyo University of Agricultural and Technology. $\mathrm{He}$ is Assistant professor of faculty of Engineering Mechanical Systems Engineering at the Tokyo University of agriculture and Technology. His primary research interests include robotics, aerospace engineering, and welfare technology.

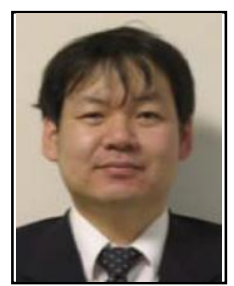

Tomozumi Ikeda was born in Matsumoto of Japan, Aug. 1, 1971. He received his Ph.D degrees from the University of Electro-Communications in 2007. He is Associate professor of faculty of human resources development at the Polytechnic University. His primary research interests include life support technology, embodied cognitive science and development of multimodal interface for visually impaired people.

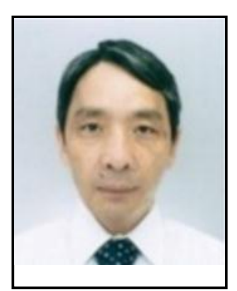

Akira Kakimoto was born in Hiroshima, Jan. 10, 1962. Graduated from the University of Tokyo in department of precision machinery engineering in faculty of engineering. Received master's degree in 1986 and doctor's degree in 1990 in department of precision machinery engineering in the graduate school of engineering in the University of Tokyo.

Research associate in department of rehabilitation engineering in the institute of vocational training (presently, the Polytechnic University) in 1990. Assistant professor in department of mechanical and control engineering in 1992. Professor in mechanical system engineering in 2010. Currently interested in assistive technology and machine control.

Prof. Kakimoto, member of EMBS IEEE, JSPE, JSME, LST, JSWSAT.

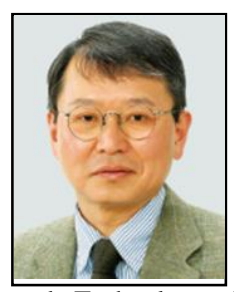

Shigeki Toyama was born in Gifu of Japan, 1954. Graduated from the University of Tokyo in department of precision machinery engineering in faculty of engineering. Received doctor's degree in 1981 in department of precision machinery engineering in the graduate school of engineering in the University of Tokyo. He is professor of faculty of Engineering Mechanical Systems Engineering at the Tokyo University of agriculture and Technology. His primary research interests include robotics, actuator, and welfare technology. 